\title{
Classifying Human-Robot Interaction: An Updated Taxonomy*
}

\author{
Holly A. Yanco \\ Computer Science Department \\ University of Massachusetts Lowell \\ One University Avenue, Olsen Hall \\ Lowell, MA 01854 USA \\ holly@cs.uml.edu
}

\author{
Jill Drury \\ The MITRE Corporation \\ 202 Burlington Road \\ Bedford, MA 01730 USA \\ jldrury@mitre.org
}

\begin{abstract}
This paper extends a taxonomy of humanrobot interaction (HRI) introduced in 2002 [1] to include additional categories as well as updates to the categories from the original taxonomy. New classifications include measures of the social nature of the task (human interaction roles and human-robot physical proximity), task type, and robot morphology.
\end{abstract}

Keywords: Human-robot interaction (HRI), taxonomy, classification of systems.

\section{Introduction}

In 2002, we introduced a taxonomy for classifying human-robot interaction [1]. In this paper, we extend the taxonomy with a number of new classifications. We have also updated the descriptions of the categories that were in the original taxonomy. This expanded taxonomy reflects changes in the field of human-robot interaction that have occurred, including research on social robots.

\section{Related Taxonomies}

Some taxonomies for human-computer interaction, robotics and human-robot interaction have already been proposed in the literature. This section describes the other taxonomies in the literature and discusses their applicability to our taxonomy.

\subsection{Computer-Supported Cooperative Work}

The canonical Computer-Supported Cooperative Work (CSCW) taxonomy is Ellis et al's "time-space taxonomy" [2]. The time-space taxonomy divides CSCW into four categories based on whether collaborators are using computing systems at the same time (synchronous) or different times (asynchronous), while in the same place (collocated) or in different places (non-collocated). For example, email systems fall into the category of asynchronous and non-collocated, while video teleconferencing systems are primarily synchronous and non-collocated. Computer-assisted crisis management spaces ("war rooms") can be used in an asynchronous yet collocated manner by teams on different shifts, while electronic meetings rooms are designed to support synchronous and collocated operation during computerassisted face-to-face meetings.

Another taxonomy of $\mathrm{CSCW}$ applications, called a "collaborative application taxonomy" [3], extends the time-space taxonomy to add a "modal" dimension: the mode of communication used by collaborators. The three modes of communication identified by Nickerson in [3] are audio, visual, or document (data); these modes may be used alone or in combination, yielding seven different possible communication approaches. Combined with the four possible time-space categories, there are 28 total categories in the collaborative application taxonomy.

The collaborative application taxonomy does not provide an adequate description of HRI because humanrobot and robot-human communication can take forms other than audio, visual, or data (for example, communication can be tactile or haptic). Nickerson's implicit assertion, that the communication mode used by collaborators is an important characteristic of collaborative systems, nevertheless has merit. In human-robot collaborative systems, communication mode is analogous to the type or means of control from the human(s) to the robot(s) and the type of sensor data transmitted (or available to be transmitted) from the robot(s) to the human(s). Thus human-to-robot control type and robotto-human sensor information type are included in our taxonomy below.

\subsection{Multi-Agent Robot Taxonomies}

Taxonomies for systems with multiple robots have also been detailed $[4,5]$, which have applicability to HRI. Dudek, Jenkin and Milios [4] point out that the task to be accomplished impacts the organization of a multi-robot team. Some of these tasks require multiple agents, as they can not be completed by a single robot. Other tasks traditionally use multiple agents, but interaction is limited. A third category is traditionally single agent tasks, meaning additional agents do not add to the speed or efficiency of the solution. Finally, there are tasks which could benefit from the use of multiple agents, although they may also be performed by a single agent. 
Balch [5] specifies a taxonomy for the "task and reward" of a multi-robot group. The task and reward taxonomy is divided into a number of categories: time (how long task is allowed, plus if synchronization is required), criteria for measuring performance (time horizon for optimization), subject of action (robot movement or object movement), resource limits (power, intra-team competition, external competition), group movement, and platform capabilities (task can be performed by a single agent, requires multiple agents, requires dispersed agents, can observe all relevant features of the world, only can get partial information, and requires communication).

Dudek, Jenkin, and Milios also use several dimensions for classifying multi-robot systems: communication range, communication topology, group size, communication bandwidth, group reconfigurability, processing ability of each group member, and group composition. This taxonomy differs from Balch by removing the task requirements explicitly from the discussion. Instead, the focus is on the composition, abilities and interactions of the group.

\section{Taxonomy Categories}

This section describes the categories in our HRI taxonomy and the classification values in each category.

\subsection{Task Type}

When discussing human-robot interaction, the task to be accomplished sets the tone for the system's design and use, so the task must be identified as part of the system's classification. The task should be specified at a high level. For example, the TASK classification could be urban search and rescue, walking aid for the blind, toy, or delivery robot. Task type also allows the robot's environment to be implicitly represented.

\subsection{Task Criticality}

Task criticality measures the importance of getting the task done correctly in terms of its negative effects should problems occur. Criticality is a highly subjective measure. To counteract this problem, we define a critical task to be one where a failure affects the life of a human. For example, the failure of a robotic wheelchair to recognize a down staircase could severely injure or kill its user. The failure of a Furby to act properly threatens no one. A hospital delivery robot does have some criticality in its task, since failure to bring a critical sample to the lab in time could be harmful. However, food delivery is a much less critical task, since a late delivery is unlikely to harm a person seriously.

Due to its subjective nature, CRITICALITY is broken into three categories: high, medium and low. Urban search and rescue has CRITICALITY=high; it is dangerous for its user to be near the disaster situation and it is important to find survivors quickly without damaging the building or hurting a trapped person. Robot soccer has CRITICALITY=low; if a robot team fails while playing another robot team, it will simply lose the game. The hospital delivery robot is an example of CRITICALITY=medium .

\subsection{Robot Morphology}

Robots can take many physical forms. We include morphology in our taxonomy because people react to robots differently based upon their appearance; "the form and structure of a robot is important because it helps establish social expectations" [6].

ROBOT-MORPHOLOGY is given three values: anthropomorphic (having a human-like appearance), zoomorphic (having an animal-like appearance), and functional (having an appearance that is neither humanlike nor animal-like, but is related to the robot's function). This division follows the morphology suggested by Fong et al [6], except that we are collapsing their category of "caricatured" (robots having simplified or stereo-typical representations) into anthropomorphic or zoomorphic, depending upon whether the caricature most closely resembles a person or an animal.

\subsection{Ratio of People to Robots}

The ratio of people to robots directly affects the human-robot interaction in a system. This taxonomy classification does not measure the interaction between the operators and the robots, simply the numbers of each. The value of HUMAN-ROBOT-RATIO is denoted as a non-reduced fraction, with number of humans over the number of robots. If the number of operators or the number of robots is variable within the system, a range may be specified in either the numerator or denominator of the ratio.

\subsection{Composition of Robot Teams}

Our taxonomy contains a classification for denoting whether robot teams contain different types of robots or the same type of robot, if there is more than one robot in use. Homogeneous teams will lend themselves to a single interface more naturally (although it may need to be repeated for each robot). To present the information from heterogeneous teams, it is likely to be more difficult to present the sensor information from different types of robots in a coherent fashion that supports decision making for each individual robot. ROBOT-TEAMCOMPOSITION can have the values homogeneous or heterogeneous. Heterogeneous may be further specified with a list containing the types of robots in the team and the number of each type of robot used in the team.

\subsection{Level of Shared Interaction Among Teams}

While the ratio of humans to robots is a distinguishing characteristic of a human-robot system, the ratio alone does not provide complete insight regarding the approach to controlling robots. The question needs to be asked: if there are multiple human controllers, are these humans agreeing on commands prior to providing the robot(s) direction, or are they independently issuing 


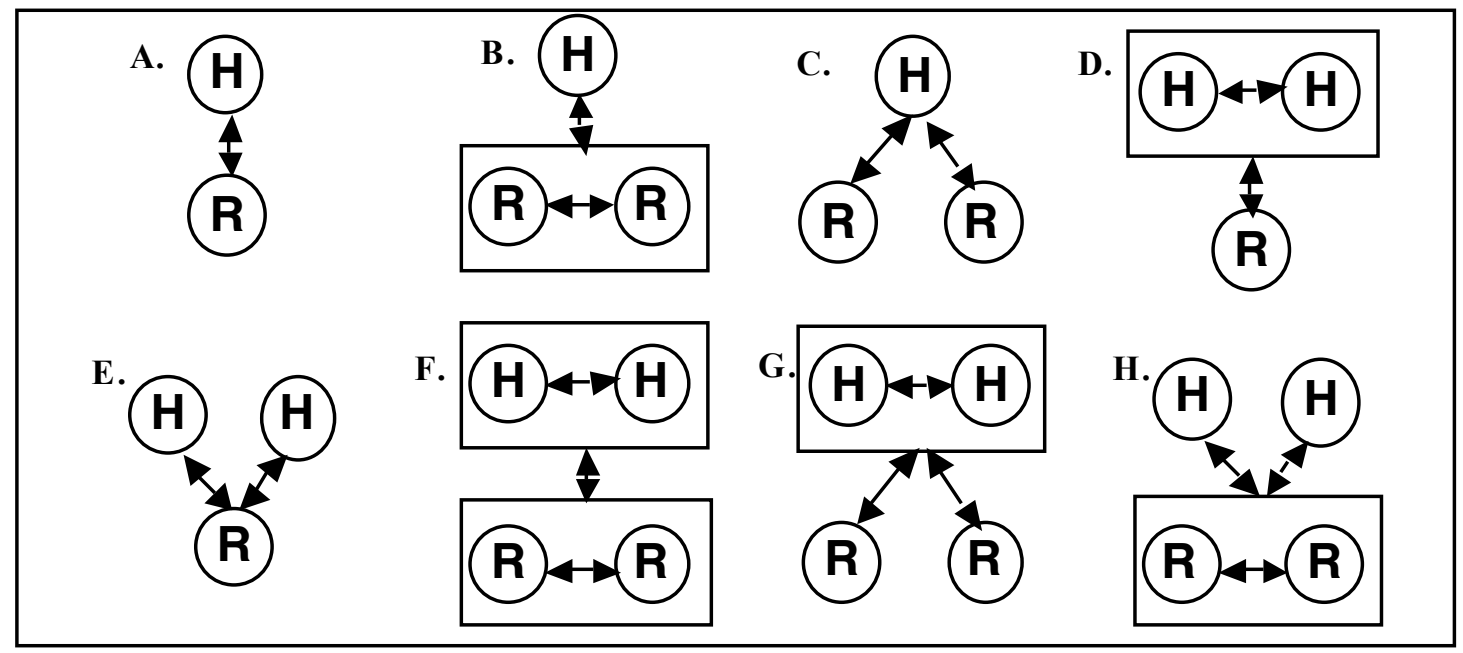

Figure 1. The possible combinations of single or multiple humans and robots, acting as individuals or in teams.

commands that $\operatorname{robot}(\mathrm{s})$ need to prioritize and/or deconflict? Also, if there are multiple robots, are they each receiving and acting on commands independently, or are all robots receiving all commands and coordinating among themselves to determine which robot(s) should respond to which commands?

In each case in Figures $1 \mathrm{~A}-1 \mathrm{H}$, a human is depicted by a circle labeled with an "H," whereas a robot is illustrated by a circle containing an "R." Doubleheaded arrows indicate command flows between the humans and robots. In the most simple case, Figure $1 \mathrm{~A}$ shows one human giving commands to one robot, which sends sensor information back to the human. An example of this case is one person directing a bomb-disposal robot. In this case, and all others discussed below, the humans need a sufficient level of human-robot HRI awareness to understand the locations, identities, activities, and surroundings of the robots they are directing. (Note that the concept of HRI awareness is defined more fully in [7].) Similarly, the robots need robot-human HRI awareness so they have knowledge of the humans' commands needed to direct their activities and any human-delineated constraints that may require command noncompliance or a modified course of action. Also, humans are always helped by a good sense of the state of current operations, which we call humans' overall mission awareness. The humans and/or robots may need other types of HRI awareness depending upon the type of teaming that may apply.

Figures $1 \mathrm{~B}$ and $1 \mathrm{C}$ show one human controlling two robots. (A maximum of two robots and two humans are shown in each figure, but the same concepts hold for "many" as for "two.") In Figure 1B, the human is giving a command to a group of robots that coordinate among themselves to determine which robot(s) should carry out which part(s) of the command. An example of this case is when a person gives a command to a group of robots to find human victims in a partially-destroyed building.
While one robot looks in one room, another robot may begin in another room, with both robots periodically comparing maps and information about victims so they avoid searching the same areas twice. These robots need robot-robot HRI awareness so that they understand enough of what their fellow robots are doing to work together most efficiently. Figure $1 \mathrm{C}$ shows one person directing two robots that work independently. This case could occur in the future in emergency services, where one person might want to direct multiple robots to different parts of a hazardous waste spill to obtain as much information about the environment as quickly as possible.

Figures $1 \mathrm{D}$ and $1 \mathrm{E}$ are the inverse of Figures $1 \mathrm{~B}$ and 1C: multiple people are controlling one robot. In Figure $1 \mathrm{D}$, the people coordinate among themselves to issue one command to the robot. An example of this situation is when a pilot and a sensor operator coordinate to fly an unmanned aerial vehicle (UAV) to a convenient spot for viewing enemy targets. These people need human-human HRI awareness, so that they understand the locations, identities, and activities of their fellow UAV team members. In Figure 1E, the humans act independently, and send different commands to the same robot. The robot must deconflict and/or prioritize the commands before carrying them out. An example of this type of robot is a waiter robot, who is asked by one person to bring canapés to a table, while another person requests that drinks are brought to another table. The robot must decide which order should be delivered first.

Figures $1 \mathrm{~F}$ through $1 \mathrm{H}$ depict the cases of multiple humans directing multiple robots. Figure $1 \mathrm{~F}$ illustrates a team of humans directing a team of robots. The humans agree on one command that the robots then coordinate on to decide what robot(s) carry out what part(s) of the command. Such a situation may occur in future UAV operations if a team of people direct a group of UAVs that coordinate so that surveillance can be maintained of the desired area despite dynamically-changing threats that 
necessitate quick rerouting. Both human-human HRI awareness and robot-robot HRI awareness are applicable in this case.

In Figure $1 \mathrm{G}$, a team of humans issues different commands to different individual robots. The humans agree which command should go to which robot, and each robot acts independently to fulfill the command (thus, no coordination is needed among robots). This situation may occur in the urban search and rescue, if multiple rescuers work together to direct individual robots to each search a different part of the destroyed area. Humanhuman HRI awareness would help the human team in this case.

Finally, Figure $1 \mathrm{H}$ shows the case where individuals do not coordinate prior to issuing different commands to a team of robots. The robots deconflict and/or prioritize the different commands as well as divide the commands among themselves prior to carrying them out. A group of industrial robots may fit into this category if they take their orders from multiple, non-coordinating humans. The robots in this case would benefit from robot-robot HRI awareness.

The taxonomy classification for the level of shared interaction among teams is INTERACTION. It can have one of the eight following values: one human, one robot; one human, robot team; one human, multiple robots; human team, one robot; multiple humans, one robot; human team, robot team; human team, multiple robots; and multiple humans, robot team. We do not include the category of "multiple humans, multiple robots," as we believe that some coordination must happen at either the human or robot end with multiple agents.

\subsection{Interaction Roles}

Scholtz [8] describes five roles that a human may have when interacting with a robot: supervisor, operator, teammate, mechanic/programmer, and bystander. One or more of these values would be assigned to the INTERACTION-ROLE classification.

A supervisory role is taken by a human when it needs to monitor the behavior of a robot, but does not need to directly control it. For example, a supervisor of an unmanned vehicle may tell the robot where it should move, then the robot plans and carries out its task.

An operator needs to have more interaction with a robot, stepping in to teleoperate the robot or needing to change the robot's behavior.

A teammate works with a robot to accomplish a task. An example of this would be a manufacturing robot that accomplished part of an assembly while a human worked on another part of the assembly of the item.

A mechanic or programmer needs to physically change the robot's hardware or software.

A bystander does not control a robot but needs to have some understanding of what the robot is doing in order to be in the same space. For example, a person who walks into a room with a robot vacuum cleaner needs to be able to avoid the robot safely.

\subsection{Type of Human-Robot Physical Proximity}

Depending upon their tasks and the purpose of the human's interactions with robot(s), robots and people may need to interact at varying interpersonal distances. In the case where humans and robots are collocated, Huttenrauch and Eklundh [9] defined five modes of physical proximity between humans and robots: avoiding, passing, following, approaching, and touching. We add "none" as a possible value for PHYSICAL PROXIMITY for the case where the robots and humans are not collocated. These values are ordered from less to more physical interaction. For cases when multiple types of physical interaction are applicable, the value chosen should be the type that involves the most physical proximity (e.g., touching rather than approaching or following).

\subsection{Decision Support for Operators}

When discussing interface design for human-robot interaction, it is most important to consider the type of information that is provided to operators for decision support. This taxonomy category has four subcategories: available sensor information, sensor information provided, type of sensor fusion, and pre-processing.

The specification of available sensors is a list of sensing types available on the robot platform; the list may also contain the location of the sensors, although this is likely to be too detailed for our requirements. The AVAILABLE-SENSORS list is used as a baseline for understanding the values of PROVIDED-SENSORS, SENSOR-FUSION, and PRE-PROCESSING; we will know what was available to the interface designer when he or she selected the sensor information to be conveyed to the operator for decision making.

The sensor information provided to the operator, PROVIDED-SENSORS, is also a list of sensing types, which is a subset of AVAILABLE-SENSORS. All of the available sensor data may not be required for decision support. For example, a robot may use its sonars to navigate, but only a video image is provided in the interface.

The type of sensor fusion, SENSOR-FUSION, is specified as a list of functions. For example, if sonar and ladar values were used to build a map that was displayed, the sensor fusion list would contain $\{$ \{sonar, ladar\} $\rightarrow$ map\}.

Finally, the amount of pre-processing of sensors for decision support is denoted in the PRE-PROCESSING list. If sonar values were used to create and display a map, the list would include $\{$ sonar $\rightarrow$ map\}. If a video stream is processed prior to display to highlight regions of a particular color, say red, the list would include \{video $\rightarrow$ highlight red regions\}.

Measuring the use of sensor data in the user interface will allow us to determine how the amount of decision support affects the performance of an operator. It should 
be easier to control a robot that provides decision support in the form of a map rather than the raw sensor readings from all of the sonar sensors at each reading, for example.

\subsection{Time/space taxonomy}

The time-space taxonomy [2] divides human-robot interaction into four categories based on whether the humans and robots are using computing systems at the same time (synchronous) or different times (asynchronous) and while in the same place (collocated) or in different places (non-collocated).

Robots such as the Mars Rover fall into the category of asynchronous and non-collocated because they are largely autonomous and are located remote from their team of human controllers. Rescue robots operate primarily in a synchronous and non-collocated manner as they explore buildings or spaces too dangerous or too small for humans to enter. Robots on the factory floor may occupy the same space (and perhaps perform the same task) as a human worker doing the same task at a later time (for asynchronous, collocated operations). Assistive robots, such as a robotic wheelchair, operate in a synchronous and collocated fashion as they are intended to help a person live better in his or her environment.

The time-space classification is specified in two values: TIME and SPACE. The possible values for TIME are synchronous and asynchronous. The possible values for SPACE are collocated and non-collocated.

\subsection{Autonomy Level / Amount of Intervention}

The amount of intervention required for controlling a robot is part of our taxonomy, because it is one of the defining factors for human-robot interaction. There is a continuum for robot control ranging from teleoperation to full autonomy; the level of human-robot interaction measured by the amount of intervention required varies along this spectrum. Constant interaction is required at the teleoperation level, where a person is remotely controlling a robot. Less interaction is required as the robot has greater autonomy.

Our definition of autonomy is very simple; for a more complete taxonomy of autonomy classes, see Huang, Messina and Albus [10]. Autonomy can also be measured by the amount that a person can neglect a system [11]

In our taxonomy, we measure the autonomy level (AUTONOMY) and amount of intervention required (INTERVENTION). The autonomy level measures the percentage of time that the robot is carrying out its task on its own; the amount of intervention required measures the percentage of time that a human operator must be controlling the robot. These two measures sum to $100 \%$.

Teleoperated robots are fully controlled by a robot operator, usually at a distance. For example, ROBONAUT [12] is a teleoperated robot that has been developed by NASA. The robot can be controlled either from a spacecraft or from the ground; its operator wears gloves to move the hands of the robot and glasses to see the robot's view of the world. ROBONAUT has AUTONOMY $=0 \%$ and INTERVENTION $=100 \%$.

At the other end of the spectrum are robots with full autonomy. Examples of this type of control can be found in robots that give tours and delivery robots. Polly [13] gave tours of the 7th floor of MIT's Artificial Intelligence Laboratory. Minerva [14] gave tours of the Smithsonian. Other robots, such as Carmel [15] and Dervish [16], could navigate indoor spaces and perform delivery tasks. All of these systems used maps of the environment and could localize themselves on the map. At this end of the continuum, the robots have AUTONOMY near 100\% and INTERVENTION near $0 \%$.

In between these two points is a large continuum of robot control, often called shared control. With shared control, the robots are able to do some part of the task and the human operator must do some part of the task. For example, the Wheelesley robotic wheelchair system [17] took over low-level navigation tasks such as path centering and obstacle avoidance in indoor and outdoor environments, while the wheelchair's user was responsible for the high-level directional commands. This wheelchair system would be classified AUTONOMY $=75 \%$ and INTERVENTION $=25 \%$.

Shared control has traditionally operated at a fixed point, where the predefined robot and operator responsibilities remain the same. However, it is easy to imagine situations where it would be desirable to have a system that could move up or down the autonomy continuum. Human operators may wish to override the robot's decisions, or the robot may need to take over additional control during a loss of communications. Research in this area has been called adjustable autonomy, sliding scale autonomy and mixed initiative. For examples of work in this area, see [18], [19], and [20]. Robots that can vary their autonomy levels would have ranges for their AUTONOMY and INTERVENTION values.

\section{Conclusions}

Drawing from the fields of $\mathrm{HCI}, \mathrm{CSCW}$, and Robotics, this paper presents an updated taxonomy for human-robot interaction. Using these classifications to define individual HRI systems will allow for the comparison of different HRI approaches in many different categories. The categories and classifications are grounded in real life examples to simplify the classification process.

\section{Acknowledgements}

This work is supported in part by NSF IIS-0308186.

\section{References}

[1] Yanco, H. A. and Drury, J. L. (2002). "A taxonomy for human-robot interaction." In Proceedings of the AAAI Fall Symposium on Human-Robot Interaction, AAAI Technical Report FS-02-03, Falmouth, Massachusetts, November 2002, pp. 111-119. 
[2] Ellis, C. A., Gibbs, S. J., and Rein, G. L. (1991). "Groupware: some issues and experiences." Communications of the ACM, 34(1): $39-58$.

[3] Nickerson, R. C. (1997). "A taxonomy of collaborative applications." In Proceedings of the Association for Information Systems (AIS) 1997 Conference, Indianapolis, IN.

[4] Dudek, G., Jenkin, M., and Milios, E. (2002). "A taxonomy of multirobot systems." In Robot Teams, Balch, T. and Parker, L.E., eds., Natick, MA: A K Peters, 2002, pp. $3-22$.

[5] Balch, T. (2002). "Taxonomies of multirobot task and reward." In Robot Teams, Balch, T. and Parker, L.E., eds., Natick, MA: A K Peters, 2002, pp. 3 - 22.

[6] Fong, T., Nourbakhsh, I., and Dautenhahn, K. (2002). A Survey of Socially Interactive Robots: Concepts, Design, and Applications. CMU-RI-TR-02-29, Technical Report from the Robotics Institute, Carnegie Mellon University, November 2002.

[7] Drury, J. L., Scholtz, J., and Yanco, H. A. (2003). Awareness in Human-Robot Interactions. In Proceedings of the IEEE Conference on Systems, Man and Cybernetics, Washington, DC, October 2003.

[8] Scholtz, J. (2003). "Theory and Evaluation of Human Robot Interactions." Hawaii International Conference on System Science 36 (HICSS 36). Hawaii, January 2003.

[9] Huttenrauch, H. and Eklundh, K. S. (2004). Investigating socially interactive robots that give the right cues and make their presence felt. Proceedings of the CHI 2004 Workshop on Shaping Human-Robot Interaction, http://www.bartneck.de/workshop/chi2004/, pp. 17 - 20.

[10] Huang, H.-M., Messina, E., and Albus, J. "Toward a generic model for autonomy levels for unmanned systems (ALFUS) PerMIS 2003.

[11] Goodrich, M. A., Crandall, J. W., and Stimpson, J. L.. "Neglect tolerant teaming: issues and dilemmas." In Proceedings of the 2003 AAAI Spring Symposium on Human Interaction with Autonomous Systems in Complex Environments, 2003.
[12] Ambrose, R. O., Aldridge, H., Askew, R. S., Burridge, R., Bluethman, W., Diftler, M. A., Lovchik, C., Magruder, D. and Rehnmark, F. (2000). "ROBONAUT: NASA's Space Humanoid." IEEE Intelligent Systems Journal, August 2000.

[13] Horswill, I. (1995). "Analysis of adaptation and environment." Artificial Intelligence, 73(1-2), pp. 1-30.

[14] Thrun, S., Bennewitz, M., Burgard, W., Dellaert, F., Fox, D., Haehnel, D., Rosenberg, C., Roy, N., Schulte, J., and Schulz, D. (1999). "MINERVA: a second generation mobile tour-guide robot." In Proc. of the IEEE International Conference on Robotics and Automation (ICRA'99).

[15] Kortenkamp, D., Huber, M., Cohen, C., Raschke, U., Bidlack, C., Congdon, C. B., Koss, F., and Weymouth, T. (1993). "Integrated mobile robot design: winning the AAAI-92 Robot Competition." IEEE Expert, August 1993.

[16] Nourbakhsh, I. (1998). "Dervish." Intelligence and Mobile Robots, Chapter 3. D. Kortenkamp, R. P. Bonasso and R. Murphy, eds. The MIT Press.

[17] Yanco, H.A. (2000). "Shared user-computer control of a robotic wheelchair system." Ph.D. Thesis, Massachusetts Institute of Technology, Dept. of Electrical Engineering and Computer Science, September 2000.

[18] Kortenkamp, D., Schreckenghost, D., and Martin, C. (2002). "User interaction with multi-robot systems." In Multi-Robot Systems: From Swarms to Intelligent Automata (Proceedings from the 2002 NRL Workshop on Multi-Robot Systems), A. C. Schultz and L. E. Parker, eds. Kluwer Academic Publishers. pp. 213 - 220.

[19] Kortenkamp, D., Keirn-Schreckenghost, D., and Bonasso, R. P. (2000). "Adjustable control autonomy for manned space flight systems." In Proceedings of the IEEE Aerospace Conference.

[20] Bruemmer, D. J., Dudenhoeffer, D. D., and Marble, J. (2002). "Dynamic autonomy for urban search and rescue." In Proceedings of the 2002 AAAI Mobile Robot Workshop, Edmonton, Canada, August 2002. 